\title{
SERVICE-ORIENTED PERFORMANCE OF INVENTORY MODELS WITH PARTIAL INFORMATION ON UNIMODAL DEMAND LEAD-TIME DISTRIBUTIONS
}

\author{
Gerrit K. Janssens, Lotte Verdonck*and Katrien Ramaekers
}

\begin{abstract}
Facing uncertainty in demand, companies try to avoid stock-outs by holding safety inventories, depending on a pre-set customer service level. The knowledge of the demand distribution during lead-time serves to determine the safety inventory level. Many times the distribution is not fully known, except maybe for its range, mean or variance. However literature shows that the performance of holding safety stock strongly depends on the characteristics of the distribution. One option is to protect against the worst case distribution given some information like range or moments. But this worst case is a two-point distribution, bringing unbelief to managers that such an occurrence would ever appear. Mostly they share the opinion that the demand distribution is unimodal. This research develops a technique to derive the safety stock for unimodal demand distributions of which the mode either is known or can be estimated. In this way, the managers obtain solutions to the decision problem including a higher belief that the related type of distribution might appear in practice.
\end{abstract}

Mathematics Subject Classification. 90B05, 90C05, 60E10.

Received May 22, 2019. Accepted March 9, 2020.

\section{INTRODUCTION AND BACKGROUND}

Inventory management has received a lot of attention both in the business world and in the academic world. Investment in inventories might be very high and the business world is confronted with fluctuations in inventories in time and with uncertainties both in demand and supply which directly influence decisions on inventories. The academic world provides managers with sophisticated systems for inventory management that enable them to take correct and timely decisions. Many decision models concentrate on the determination of inventory replenishment quantities based on relevant costs like order costs or storage costs. Most of these models assume deterministic demand patterns. But in real life uncertainties appear both in demand, as in supply or even in the quality of the delivered goods. This research investigates in detail a specific case of inventory management decisions in the case of demand uncertainty.

In inventory management decisions on order quantities and safety stocks are made on the basis of optimisation models taking a performance measure into consideration which might be cost-oriented or service-oriented. Cost-oriented measures take into consideration costs related to a customer's order when the item ordered is

Keywords. Inventory management, linear programming, partial information, demand distribution.

Research Group Logistics, Hasselt University, Diepenbeek, Belgium.

*Corresponding author: lotte.verdonck@uhasselt.be 
temporarily out of stock. Two extreme policies are proposed in the scientific literature: complete back ordering or complete lost sales, but in practice many intermediate policies may be applied. In the "complete backordering" policy, demand, when out of stock, is backordered and filled as soon as an adequate-size replenishment arrives. In the "complete lost sales" case, demand, when out of stock, is lost. The customer goes elsewhere to satisfy the need [30]. In both policies a cost is related to the stock-out. In the former case, it leads to additional transport costs, additional paper work, and a delay in payment. In the latter case, it leads to an opportunity cost as lost sales lead to lost profits. Performance measures of the service-oriented type may be expressed relatively as a probability of a stock-out during a certain replenishment period, or may be expressed absolutely in terms of the expected number of units short, which is a direct indication for lost sales. These types of measures do not explicitly include a cost for additional paperwork or transport cost or loss of goodwill as, in practice, experience shows that these costs are hard to determine.

Both in the cost-oriented as in the service-oriented performance evaluation, a specific integral plays an important role in the decision making. This integral is defined by [30] as the expected shortage per replenishment cycle (ESPRC):

$$
\operatorname{ESPRC}=\int_{t}^{+\infty}(x-t) f(x) \mathrm{d} x
$$

in which it is assumed that the demand $X$ in a replenishment lead time has a probability density function $f(x)$ and an order is placed at some time when the inventory position is at or below level $t$.

If ordered per quantity $Q$, the fraction backordered is equal to $\operatorname{ESPRC} / Q$ and a performance measure, indicated as $P_{2}$, is defined as:

$$
P_{2}=1-\mathrm{ESPRC} / Q \quad(\text { see }[30], \text { p. 299). }
$$

Consider the case of a complete backordering policy. As each replenishment is of size $Q$, the fraction backordered is ESPRC $/ Q$, and the fraction of demand satisfied directly from shelf is $1-\operatorname{ESPRC} / Q$. So, $P_{2}$ is a measure in the case of backordering. In the case of lost sales, the alternative service measure is needed. That is because the demand per replenishment cycle is not just $Q$ but $Q+\operatorname{ESPRC}$. The service measure "fraction of demand satisfied directly from shelf" is rather $1-\operatorname{ESPRC} /(Q+\mathrm{ESPRC})$.

While this study concentrates on service-oriented performance, also in cost-oriented types of performance measures the integral (1.1) is of high relevance. This is illustrated in two examples.

Example 1.1. In a study by [11] a $(Q, t)$ inventory system with backordering is studied. In such a policy a fixed order quantity $Q$ is ordered in case the inventory position reaches a level $t$ or below. The objective is to determine values of $Q$ and $t$ so that the long run average of the total of ordering, storage and backordering costs are minimised. The long run average cost function equals:

$$
\text { Cost }=\frac{K D}{Q}+h\left(\frac{Q}{2}-t-\mu\right)+\frac{\pi D}{Q} \int_{t}^{+\infty}(x-t) f(x) \mathrm{d} x,
$$

in which $K=$ the fixed order cost, $D=$ the average demand per period, $h=$ the unit storage cost per period, $\mu=$ the mean lead-time demand, $\pi=$ the unit shortage cost, and $F(x)$ is the cumulative distribution of the lead-time demand with density function $f(x)$. The last term in the cost function (1.3) corresponds to the earlier defined performance measure in equation (1.1).

Example 1.2. Another example relates to the newsboy problem, which symbolises a single-period inventory problem. A decision has to be made on a single purchase before the start of the selling period and can be used only to satisfy the demand during that period. The demand is random. The trade-off relates to overstocking forcing the disposal to sell at very low price or understocking losing the opportunity to make a profit. The relevant costs can be determined on the basis of the ending inventory, making use of $k_{o}$, which is the cost per unit of positive inventory remaining at the end of the period (known as overage cost), and $k_{u}$, which is the cost 
per unit of unsatisfied demand (known as underage cost) [25]. The value of the expected cost $\Phi$ of the decision problem, for a demand distribution with density function $f(x)$ and a fixed order quantity $Q$ is given by:

$$
\Phi(Q)=\int_{0}^{Q} k_{o}(Q-x) f(x) \mathrm{d} x+\int_{Q}^{\infty} k_{u}(x-Q) f(x) \mathrm{d} x .
$$

Other formulations of equation (1.4) exist, of which one is specifically of interest to this study. It is given by [28] as:

$$
\Phi(Q)=k_{o}(Q-\mu)+\left(k_{o}+k_{u}\right) \int_{Q}^{\infty}(x-Q) f(x) \mathrm{d} x,
$$

where $\mu$ is the expected value of the demand distribution. This formula is used to obtain optimal values in a $(R, S)$-policy stochastic production/inventory control problem. An $(R, S)$ policy is a periodic review policy in which the inventory level is only observed at intervals of length $R$. If the inventory is at level $y$, a quantity $S-y$ is ordered to bring the inventory position to $S$. Explicit formulas are obtained in the case the demand in the single-period follows a normal distribution.

While the linear programming approach, which has been used before in [18], is also in this research the main technique, the formulation of the constraints is different in the case of knowledge of the mode. While it is definitely not classical in inventory decision-making to make use of the mode of a distribution instead (or as a surplus to) the variance, it is innovative and the solution is less confronting in comparison with the two-point distribution for the worst-case distribution without knowledge of the mode. Of course, such an innovation leads to the question which information, "variance" or "mode", is of higher value. This question is also touched in this research. In a real-life situation with possibly thousands of stocked items in the warehouse, it might be questioned what the additional value is of a computational effort for solving linear programs, which is definitely higher than using the normal distribution approach with the estimates of mean and variance of the demand distribution. It is our advice that the approach, as introduced here, might be useful and cost-saving in two cases: (1) in the case in which, from observation of historical data, it can be concluded that the shape of the distribution is far from a normal distribution, being either very skewed or bimodal; and (2) in the case where stock-outs are commercially important in terms of service or cost, and the company wants to avoid a stock-out at all price. For items with a low service level target, the solution of the linear program might not add any additional value. Also the use of the upper bound is most probably too conservative for this type of items.

The main contribution of this article is the development of a linear program, which can be used by an inventory manager to decide on the reorder point. In contrast to the worst case solution with respect to holding a safety stock, which is a two-point distribution, this approach leads to a solution which fits more to the knowledge in the mind of the inventory manager as they mostly experience a demand distribution to be unimodal, even if the mode of this distribution is not exactly known.

Example 1.3. It is assumed that the demand for a product lies in the range $[0,50]$ during the lead-time period. Furthermore assume that the mean equals 35 and the standard deviation equals 20 . The worst case for the measure "expected number of units short" is realised when the demand is a two-point distribution located in the points 8.3 and 50 , with respective probabilities 0.36 and 0.64 . In most cases, the manager would probably tell that the product definitely has not this type of discrete distribution, defined in only two points.

In the following sections the methodology for obtaining solutions to the direct and inverse problems is presented. First, in Section 2, the relevant literature is reviewed. In Section 3, the case with known range, first and second moments is highlighted. In Sections 4 and 5, the case of unimodal distributions with known unique mode, range, first and second moments is studied. Section 6.1 offers a solution for the inverse problem. In the case of unimodal demand distributions, it is assumed that the value of the unique mode is exactly known. In practice only an expert opinion or a statistical estimate of the mode may be available. The sensitivity of the results with respect to the uncertainty on the value of the mode is studied in Section 6.2. The sensitivity of the results with respect to the uncertainty on the value of the upper bound of the demand interval is investigated 
in Section 6.3. Section 7 illustrates how the methodology would work in a real-life environment. Simulated data for demand during a number of periods are generated. From the data set the parameters for the methodology are obtained (range, moments and mode). With this information, our method is further applied.

\section{LiterATURE REVIEW}

This research aims to warn a person responsible for inventory management for the impact of the probability distribution of the demand during lead-time on the performance of his/her decisions. Numerous simulation studies have supported this warning. These studies stress the fact that, in case the functional form of the distribution is not (fully) known, the common assumption of making use of the normal distribution might be very harmful. Naddor [24] finds that false assumptions about the distribution may lead to higher cost in the case of extreme distributions, but that, with realistic distributions, only the first and second moments are essential. On the other side, Bartezzaghi et al. [1] show a significant impact of the shape of the demand distribution on the service level, based on a large set of experiments. Their analysis shows that the shape is a primary factor in the determination of inventories and that the impact of different demand shapes on inventories is comparable to the effect of doubling the coefficient of variation of the demand distribution. Furthermore, Käki et al. [20] show the impact of the demand distribution shape on replenishment, based on experiments with qualitative shape characteristics (normal, positively skewed, negatively skewed, and bimodal). For example, they find that the safety stock requirement of positively skewed demand distribution is $137 \%$ higher compared to negatively skewed demand distributions, with identical expected value and variance. Heuts et al. [15] investigate the influence of skewness and kurtosis on the inventory decisions, by making using of the Schmeiser-Deutsch class of distributions. This class of distributions is well-suited for solving problems in operations research or industrial engineering where distributions are required with specified values of skewness or kurtosis. Given this information, the distribution needs four parameter values to generate variables (for simulation) or to use as an approximate approach to known distributions. They show that asymmetry of the demand distribution has a large effect on the inventory decisions and, by this, also on the cost. It could be even an unreasonable assumption that the demand obeys a known distribution for several types of agricultural products. In such a case an inventory replenishment policy has been proposed on the mean of the distribution only [4]. Scarf et al. [29] has done pioneering work for a robust solution of the news-vendor problem where the probability distribution of the demand is ambiguous (read: only first and second moments are known). His approach is to find the worst-case solution and make the decision to avoid this case. Perakis and Roels [26] decide on order quantities for the news-vendor problem, not from a viewpoint of the worst-case solution, but from a viewpoint of maximum regret of not acting optimally. In [18] an approach has been developed to obtain the reorder point based on the knowledge of the mean and variance of the demand distribution, which is the same information as required for the use of the normal distribution (as many times used in commercial software). The approach is a worst-case approach, which means that its solution protects the decision-maker against the worst distribution with the same mean and variance, as used in the normal distribution approach. This means that, even in the worst case distribution assumption, the pre-set service level is obtained. Table 1 gives an overview of a number of approaches, as discussed in the literature review, to handle cost-oriented or service-oriented inventory decision-making in which full knowledge of demand distributions are confronted with their partial information counterparts. Also a few non-probabilistic approaches are included in the table.

Demand forecasting and inventory management are inevitably connected. While this connection might be recognised by practicians, it can be observed that the academic literature has shown little interest and, even if some early articles on this matter have appeared long ago [3]. Also recently some authors claim that the connection has not been investigated in a sufficient way [32].

In the last decade some considerable effort has been paid to slow-moving items, more especially those with intermittent demand. Long after Croston's work [5], a lot of interest has been shown and methods have been developed and have been refined for items, which have zero demand in some time periods [31]. 
TABLE 1. Overview of approaches of inventory decision models using partial information of the distribution of the demand during lead-time.

\begin{tabular}{|c|c|c|c|}
\hline Author & Partial information & Distributions & Cost or service \\
\hline Naddor $[24]$ & Mean, variance, range & $\begin{array}{l}\text { Poisson, Beta, Uniform, Nega- } \\
\text { tive binomial, Two-point }\end{array}$ & $\begin{array}{l}\text { Cost function for }(s, S) \text {-policy by discrete } \\
\text { dynamic programming }\end{array}$ \\
\hline Bartezagghi et al. [1] & Coefficient of variation & $\begin{array}{l}\text { Uniform, Normal, Beta, Bi- } \\
\text { modal }\end{array}$ & Service level (percentage of satisfied demand) \\
\hline Käki et al. [20] & Mean, variance & $\begin{array}{l}\text { Normal, Positive skewed, Neg- } \\
\text { ative skewed, Bi-modal }\end{array}$ & $\begin{array}{l}\text { Cost function (facility, capacity, transporta- } \\
\text { tion, shortage) for }(Q, R) \text {-policy }\end{array}$ \\
\hline Chen et al. [4] & Constant demand rate & No distribution used & $\begin{array}{l}\text { Cost function (holding, order, deterioration, } \\
\text { purchase) by system dynamic model }\end{array}$ \\
\hline Janssens and Ramaekers [18] & Mean, variance, range & Distribution-free & $\begin{array}{l}\text { Service level (units short) by linear program- } \\
\text { ming }\end{array}$ \\
\hline Scarf $[29]$ & Mean, variance & Distribution-free & $\begin{array}{l}\text { Profit function (maximize minimum profit) } \\
\text { by analytical solution }\end{array}$ \\
\hline Perakis and Roels [26] & $\begin{array}{l}\text { Mean, variance, mode, } \\
\text { range }\end{array}$ & $\begin{array}{l}\text { Truncated normal, Gamma, } \\
\text { Log-normal, Negative binomial }\end{array}$ & Minimax regret for news-vendor problem \\
\hline Heuts et al. [15] & $\begin{array}{l}\text { Mean, variance, skew- } \\
\text { ness and kurtosis param- } \\
\text { eters }\end{array}$ & Schmeiser-Deutsch & $\begin{array}{l}\text { Cost function (per unit, purchase, holding, } \\
\text { shortage) for the }(s, q) \text {-model }\end{array}$ \\
\hline Dey and Chakraborthy [8] & Triangular fuzzy number & No distribution: fuzzy demand & $\begin{array}{l}\text { Cost function in the fuzzy sense for the peri- } \\
\text { odic review control system }\end{array}$ \\
\hline Bertsimas and Thiele [2] & Mean, variance & Gamma, Log-normal, Normal & $\begin{array}{l}\text { Cost function over finite period (order, hold- } \\
\text { ing, shortage) by robust linear programming }\end{array}$ \\
\hline Li et al. [22] & $\begin{array}{l}\text { Single point or interval } \\
\text { forecast }\end{array}$ & $\begin{array}{l}\text { No distribution: single point or } \\
\text { interval transformed in belief } \\
\text { distribution }\end{array}$ & $\begin{array}{l}\text { Cost function (purchase, holding, shortage) } \\
\text { by belief-rule-based inference methodology }\end{array}$ \\
\hline This research & $\begin{array}{l}\text { Mean, variance, range, } \\
\text { mode }\end{array}$ & Distribution-free & $\begin{array}{l}\text { Service level (units short) by linear program- } \\
\text { ming }\end{array}$ \\
\hline
\end{tabular}

In a stable business environment, the demand probability distribution can be estimated using historical data. Historical data may be not or not sufficiently available in a dynamic business environment, after introduction of recent products on the market, or in the case of slow moving items. In some cases, first and second moments of the distribution might be calculated, but no further statement can be made on the type of demand distribution. Sometimes one must rely on domain experts to estimate the demand probability distribution, typically through its mean and variance.

In both cases, one should be aware of the errors of the estimation. In the former case because of the small number of statistical data, in the latter case because of judgmental errors. Empirical evidence exists that experts do not perform well in providing subjective estimates on mean and variance. Zapata-Vazquez et al. [33] show that poor estimates for the mean may come out of expert judgments, but Garthwaite et al. [12] show that there exist "misconceptions about variance" and mostly it is underestimated, because the physical meaning of a variance is difficult to grasp.

It should be noted that also non-probabilistic inventory control strategies have been formulated as valid alternatives. Amongst these alternatives fuzzy mathematical programming [8], robust optimisation [2] and a belief-rule-based inference methodology [22] need to be taken into consideration.

In case only partial information on the probability density function is available (like range, mean or variance), we determine the worst-case distribution. The mathematical development, however, shows that the worst-case distributions (in case mean and variance are known) are discrete distributions in which the mass is concentrated in two points, with at least one point located at a boundary of the finite range. This property has already been described by [29]. The presentation of such a solution to a manager could lead to an expression of unbelief, stating that such a type of demand distribution does not appear in the practical case under study. At the end of this section, this statement is explained in more detail by means of an example. A most typical explanation for this unbelief is that the distribution is not bimodal, but has a single mode such as the normal distribution or skewed distributions like the Gamma-distribution. Such a statement certainly does not imply that bimodal distributions for demand in a lead-time period do not appear in practice but they are not common. 
But the fact that the distribution should have a shape with a single mode, has motivated this study to investigate what influence this additional information has on the determination of worst-case distributions.

\section{A guideline on how to handle the DeCision PROBlem With incomplete INFORMATION ON DEMAND}

In this section, an example is worked out to illustrate how the problem needs to be solved. It will be illustrated that the direct problem leads to a linear programming problem in determining the value of the performance measure but that the decision problem involves a set of nonlinear constraints to deal with. This section shows how to formulate the linear program to obtain the value of the direct problem and how to handle the nonlinear constraints in the decision problem. The current section is based on material which appeared in [18]. The following section extends this idea in the context of unimodal demand distributions.

In this section, the partial information on the demand during lead time includes the finite support of the distribution, and the first and second moments. Let the size of the demand $X$ for a specific product in a finite period have a distribution $F$ with first two moments $m_{1}=E(X)$ and $m_{2}=E\left(X^{2}\right)$. The distribution $F$ is defined on the finite interval $[a, b]$. Without loss of generalisation, the development is continued with the demand during lead-time defined on the interval $[0, b]$.

From a mathematical point of view, the problem is to find the following bounds:

$$
\sup _{F \in \phi} \int_{0}^{\infty}(x-t)_{+} \mathrm{d} F(x)
$$

and

$$
\inf _{F \in \phi} \int_{0}^{\infty}(x-t)_{+} \mathrm{d} F(x)
$$

where $(x-t)_{+}$stands for $\max (x-t, 0), \phi$ is the class of all distribution functions $F$ which have first and second moments $m_{1}$ and $m_{2}$, and which have support in $\Re^{+}$. Let further $\sigma^{2}=m_{2}-m_{1}^{2}$. We assume $t$ to be strictly positive.

In this section, we consider the supremum version of the relevant integral in equation (3.1). Let $f_{1}, f_{2}, \ldots, f_{n}$ be functions on $\Re$. Functions $f_{1}, f_{2}, \ldots, f_{n-1}$ are used to obtain the moments of order 1 till $n-1$ and function $f_{n}$ stands for the number of units short. For any $z^{\prime}=\left(z_{1}, \ldots, z_{n-1}\right) \in \Re^{n-1}$, we consider the primal maximisation problem:

$$
P\left(z^{\prime}\right)=\sup _{F \in \phi}\left[\int_{0}^{\infty}(x-t)_{+} \mathrm{d} F(x) \mid I(F)\right],
$$

where $I(F)$ is a set of integral equality constraints of the type $\int f_{i}(x) \mathrm{d} F(x)=z_{i},(i=1, \ldots, n-1)$ and $f_{n}=(x-t)_{+}$. In our application, the constraints are moment constraints, i.e. the first and second moment equalities and the obvious constraint because any member of $\phi$ is a probability distribution.

$$
\int \mathrm{d} F(x)=1, \int x \mathrm{~d} F(x)=m_{1}, \int x^{2} \mathrm{~d} F(x)=m_{2},
$$

which means that:

$$
\begin{aligned}
n & =3 \\
f_{1}(x) & =x \\
f_{2}(x) & =x^{2} \\
f_{3}(x) & =(x-t)_{+} \\
z_{1} & =m_{1} \\
z_{2} & =m_{2} .
\end{aligned}
$$


The extreme distributions for both supremum and infimum problems have been shown to be two-point or three-point distributions [7]. The integral may be approximated by a sum making use of finite masses $p_{i}$ in a larger number of points $x_{i}$. Its formulation looks like:

$$
\operatorname{Max} \sum_{i}\left(x_{i}-t\right)_{+} \times p_{i}
$$

subject to

$$
\begin{aligned}
\sum_{i} p_{i} & =1, \\
\sum_{i} x_{i} \times p_{i} & =m_{1}, \\
\sum_{i} x_{i}^{2} \times p_{i} & =m_{2},
\end{aligned}
$$

and $p_{i} \geq 0,0 \leq x_{i} \leq b$. However, any refinement in granularity leads to an increased number of variables both in the objective function (3.5) and in the three constraints (3.6) to (3.8). This phenomenon does not guarantee any convergence towards the exact upper bound. This is because in each subsequent linear program, by increasing the number of points $x_{i}$, the set of decision variables $p_{i}$ changes. Also the constraints (3.6) to (3.8) in subsequent linear programs have no link with their predecessors. The optimisation problem (3.3) and (3.4) has a dual program of the type:

$$
\begin{array}{r}
Q\left(z^{\prime}\right)=\inf \left(y_{1} m_{1}+y_{2} m_{2}+y_{3} \mid y_{1} \widehat{f}_{1}(\theta)+y_{2} \widehat{f}_{2}(\theta)+y_{3} \geq \widehat{f}_{3}(\theta)\right) \\
(\theta \in J),
\end{array}
$$

where the infimum is over all $y=\left(y_{1}, y_{2}, y_{3}\right) \in \Re^{3}$ satisfying the constraints indicated after the slash. The functions $\widehat{f}_{i}(\theta)(i=1, \ldots, n)$ are defined on $J$. In the situation for the family of distributions on a finite interval $[0, b]$, where $b$ is a fixed positive number, the three functions are [13]:

$$
\widehat{f_{1}}(\theta)=\theta, \quad \widehat{f_{2}}(\theta)=\theta^{2}, \quad \widehat{f_{3}}(\theta)=(x-\theta)_{+} \quad(0 \leq \theta \leq b) .
$$

Mostly the size of the set $J$ is infinite, so the number of linear constraints on $y$ is infinite. In [13] an idea is launched to replace $J$ by a large finite subset of $J$ and then to solve the so obtained linear program. Through a careful selection of the $\theta$-values convergence to the supremum is guaranteed as exemplified by [17]. This is because subsequent linear programs look very similar. The set of decision variables remains the same. Furthermore, all constraints from the predecessor programs remain. More constraints are added, which is pushing the approximate solution to the real solution. The optimisation problem (3.9) can thus be approximated by the problem $Q^{A}$ :

$$
\begin{array}{r}
Q^{A}\left(z^{\prime}\right)=\inf _{y \in \Re^{3}}\left(y_{1} m_{1}+y_{2} m_{2}+y_{3} \mid y_{1} \theta_{i}+y_{2} \theta_{i}^{2}+y_{3} \geq\left(\theta_{j}-t\right)_{+}\right), \\
\left.\left(\theta_{j}=j \times \frac{b}{k}, j=0, \ldots, k\right)\right) .
\end{array}
$$

A numerical example is taken from [19] to illustrate how the approximation method works. It aims at understanding more easily the case under study in this research, i.e. the case of unimodal distributions. Also the way how to formulate and how to solve the inverse problem is based on [19].

Example 3.1. Assume a demand distribution defined on a finite interval $[0,50]$, with first and second moments $m_{1}=25$ and $m_{2}=725$. The upper bounds for various values of $t$ and $k$ are shown in Table 2 . The exact values for the upper bounds, obtained with a very large number of constraints, are 16.37931 for $t=10,5.0$ for $t=25$ and 1.37931 for $t=40$. 
TABLE 2. Upper bounds on the expected number of units short.

\begin{tabular}{rrrrr}
\hline \hline & $k=10$ & $k=20$ & $k=40$ & \multicolumn{1}{c}{$k=80$} \\
\hline$t=10$ & 16.3333 & 16.3636 & 16.3768 & 16.3784 \\
$t=25$ & 5.0000 & 5.0000 & 5.0000 & 5.0000 \\
$t=40$ & 1.3333 & 1.3636 & 1.3768 & 1.3784 \\
\hline
\end{tabular}

Let us call this problem "the direct problem", as it calculates the upper (or lower) bounds on the expected number of units short, given the partial information on the DDLT (in Example 3.1, partial information means range, first and second moments). But from a management point of view, it is of interest to determine the value of $t$ to meet a pre-set service level (in this expressed as the expected number of units short in a lead-time period). Similar to the approximation, as used in formula (3.11), a method will be worked out in which the objective function is minimized satisfying a set of constraints evaluated in a discrete number of points $x_{i}(i=1 \ldots k)$. This approach leads to the solution of the continuous problem if $k \rightarrow \infty$.

This leads to an optimisation problem, where:

$t=$ the reorder point

$p_{i}=$ the probability mass in point $x_{i}$

$z_{1}=$ the expected value

$z_{2}=$ the absolute second moment

$z_{3}=$ the maximum allowed expected number of items short.

The optimisation problem [P1] might be formulated as:

$$
[\mathrm{P} 1] \operatorname{Min} t \text {, }
$$

subject to

$$
\begin{aligned}
\sum_{i} p_{i} & =1, \\
\sum_{i} x_{i} \times p_{i} & =z_{1}, \\
\sum_{i} x_{i}^{2} \times p_{i} & =z_{2}, \\
\sum_{i}\left(x_{i}-t\right)_{+} p_{i} & \leq z_{3},
\end{aligned}
$$

where $\left(x_{i}-t\right)_{+}$stands for $\max \left(x_{i}-t, 0\right)$ and $x_{i}$ takes values $(i \times b) / k$ for $i=1 \ldots k$. The decision variables in [P1] are $t$ and $p_{i}(i=1 \ldots k)$, where $k$ represents the number of discrete points which have been chosen in the experiment.

The non-linear constraint (3.16) may be approximated by letting the value of $t$ coincide with one of the $x_{i}$ values (so as $k \rightarrow \infty$, the approximation takes the correct value). In such a way, the constraint is linearised.

In the case $t$ coincides with a point $x_{j}$ then

$$
\sum_{i=1}^{n} p_{i}\left(x_{i}-x_{j}\right)_{+} \leq z_{3} .
$$

A binary variable needs to be introduced to indicate the condition " $t=x_{j}$ ". In the case $t$ does not coincide with a point $x_{j}$, a general truth should be indicated, for example, "the expected number of units short cannot 
TABLE 3. Minimal required inventory level based on the maximum expected number of units short.

\begin{tabular}{lllll}
\hline \hline & $k=10$ & $k=20$ & $k=40$ & $k=80$ \\
\hline$z_{3}=2$ & 25.0000 & 25.0000 & 25.0000 & 25.0000 \\
$z_{3}=4$ & 25.0000 & 22.5000 & 21.2500 & 21.2500 \\
$z_{3}=6$ & 20.0000 & 20.0000 & 20.0000 & 19.3750 \\
\hline
\end{tabular}

be larger than the expected demand", expressed by a binary variable $y_{j}$ :

$$
\begin{aligned}
y_{j} & =1 \text { if } t=x_{j}, \\
& =0 \text { if else. }
\end{aligned}
$$

As $t$ can coincide with only one $x_{j}$ value, the additional constraint is introduced:

$$
\sum_{j=1}^{n} y_{j}=1 .
$$

The $y$ variable is introduced in constraint (3.17) as:

$$
\sum_{i=1}^{n} p_{i}\left(x_{i}-x_{j}\right)_{+} \leq z_{3} y_{j}+z_{1}\left(1-y_{j}\right) .
$$

Finally, a link should be made between $t$ and the value of $x$ with which $t$ coincides.

$$
t \geq x_{j} y_{j} \quad \forall j
$$

If $y_{j}=0$, a universal truth is mentioned.

Example 3.2. The elaboration above will be illustrated by means of a numerical example similar to the one demonstrated above. With the support of the demand distribution equal to $[0,50]$, an expected value $z_{1}=25$ and a second moment $z_{2}=725$, Table 3 presents the minimal amount of inventory for different values of $z_{3}$ (maximum expected number of items short) and varying sizes $k$ of the evaluation point set. The exact values, obtained with a very high value of $k$, for the minimal inventory level are 25 for $z_{3}=2,21$ for $z_{3}=4$ and 19 for $z_{3}=6$.

Note that in experiments, like the one in Table 3, subsequent increasing values of $k$ need to include all constraints from its previous values to ensure convergence.

\section{The CASE OF UNIMOdAL LEAD-TIME DEMAND DistRIBUtions}

Sometimes it is considered difficult to observe or to estimate both first and second moments, but experts have an opinion on the unimodality of distributions, either or not with some additional knowledge on the expected value. The estimation of the mode of a distribution, be it in a subjective manner or via limited data, for use in inventory management, is not classical. But the use of an estimate of the mode has been common in expert opinion on the duration of activities in project planning, where experts estimate three values: an optimistic, a most likely (or modal) and a pessimistic duration [23,27]. More applications on three-point approximations of probability distributions can be found in [21]. We are not aware of any studies investigating the quality of a subjective judgment of the mode. This type of studies has been done for accuracy on estimation of the mean and variance only [16]. In this case, we consider how to handle this type of partial information, including 
knowledge of the mode, to obtain bounds on the performance measure under study. First, some additional definitions are introduced and afterwards it is shown how this problem can be transferred into the one described in Section 2.

Let $I$ be an interval on the real line $\Re$. A fixed point $m \in I$ is called the mode. For $\alpha \in I$, we denote by $1_{\alpha}$ the indicator function of the closed interval with extremities $m$ and $\alpha$. Thus $1_{\alpha}=1_{[\alpha, m]}$ if $\alpha \leq m$ and $1_{\alpha}=1_{[m, \alpha]}$ if $\alpha \geq m$.

A m-unimodal density function is a density function increasing (not necessarily strict) at the left of $m$ and decreasing (not necessarily strict) at the right of $m$. The convolution of two symmetric unimodal distributions on $\Re$ is symmetric and unimodal. A linear combination with positive coefficients of $m$-unimodal densities is a $m$-unimodal density, with the positive coefficients summing up to 1 . In particular, $1_{\alpha}$ is a $m$-unimodal density and any linear combination with positive coefficients of such indicator functions is a $m$-unimodal density.

The problem of finding extreme values for the performance measure "expected number of units short" will be transformed to the problem in the previous section by making use of a probabilistic version of a formal criterion by Khintchine $[9,10]$. It states that a stochastic variable $Z$ is unimodal with mode at the origin 0 and domain $I$ if and only if $Z$ has the same distribution as the product $U V$ of two independent variables such that $U$ is distributed uniformly in $[0,1]$ and $V$ is defined on the domain $I$.

If the lead-time demand $X$ is defined on $[0, b]$ with mode located at $m$, then the theorem can be applied for a variable $Z=X-m$ and the variable $V$ is defined on $[-m, b-m]$. In this case it is true for every function $f$ that $E[f(Z)]=E\left[f^{*}(V)\right]$ where

$$
\begin{aligned}
f^{*}(x) & =E[f(U V) \mid V=x], \\
& =\frac{1}{x} \int_{0}^{x} f(\tau) \mathrm{d} \tau .
\end{aligned}
$$

The theorem in equation (4.1) is explained here. For a rigorous proof, the reader should consult ([10], p. 158). Let $X$ and $Y$ be independent random variables with distributions $F$ and $G$, and assume that $X>0$. The product $Z=X Y$ has a distribution function $U$ of $Z$ by integrating

$$
P(Z \leq t \mid X=x)=G\left(\frac{t}{x}\right)
$$

More specifically, if $X$ is distributed uniformly over $[0,1]$, then

$$
U(t)=\int_{0}^{1} G\left(\frac{t}{x}\right) \mathrm{d} x .
$$

Choose a value $h>0$ and denote $U_{h}$ the distribution function which agrees with $U$ at the points $0, \pm h, \pm 2 h, \ldots$, so $U_{h}(n h)=U(n h)$ and $U_{h}$ is linear between $n h$ and $(n+1) h$. This means that $U_{h}$ has a density $u_{h}$, which is a step function with discontinuities at the points $n h$. The step function can be written as

$$
\sum p_{n} \frac{1}{|n| h} f\left(\frac{x}{n h}\right)
$$

where $f(x)=1$ for $0<x<1$ and $f(x)=0$ elsewhere. The function is monotone in $(-\infty, 0)$ and in $(0,+\infty)$ if $p_{n} \geq 0$ for all $n$. It is a probability density if $\sum p_{n}=1$. Note that $U$ is unimodal if all $U_{h}$ are unimodal. But now the function can be written as $Z_{h}=X Y_{h}$ where $X$ is distributed uniformly in $(0,1)$ and $P\left\{Y_{h}=n h\right\}=p_{n}$. That means $U_{h}$ is unimodal with $G$ replaced by an arithmetic distribution $G_{h}$ (concentrated on the points $0, \pm h, \pm 2 h, \ldots)$. By letting $h \rightarrow 0$, the proof of the theorem is obtained.

Let us define $Y=V+m$. Then $Y$ is defined without further restrictions on $[0, b]$ and $E[f(X)]=E[g(Y)]$ where:

$$
g(x)=\frac{1}{x-m} \int_{0}^{x-m} f(\tau+m) \mathrm{d} \tau .
$$


In the case of the function $f$ representing the expected number of units short, one has $f(x)=(x-t)_{+}$, so this means:

If $m<t$ :

$$
\begin{aligned}
& \text { If } x<m: \\
& \qquad g(x)=\frac{1}{m-x} \int_{m-x}^{0}(\tau+m-t)_{+} \mathrm{d} \tau=0 .
\end{aligned}
$$

If $m<x<t:$

$$
g(x)=\frac{1}{x-m} \int_{0}^{x-m}(\tau+m-t)_{+} \mathrm{d} \tau=0
$$

If $t<x \leq b$ :

$$
g(x)=\frac{1}{x-m} \int_{t-m}^{x-m}(\tau+m-t)_{+} \mathrm{d} \tau=\frac{1}{2} \frac{(x-t)^{2}}{x-m}
$$

which can be written, for $0 \leq x \leq b$ and $x \neq m$ as:

$$
g(x)=\frac{1}{2} \frac{\left((x-t)_{+}\right)^{2}}{x-m} .
$$

The case $m>t$ is not taken into consideration here. This means, for example in the case of symmetric demand distributions, that a negative safety stock would be held. For the greater part of the products, such a policy does not make sense.

In the remaining part of this paper, the application of the results will be illustrated for two problems, which we call the direct problem and the inverse problem. The direct problem obtains upper and lower bounds for the performance measure "expected number of units short", given a reorder point $t$. The inverse problem obtains upper and lower bounds for the reorder point $t$ given a maximum value for the expected number of units short. Both problems are solved in two cases which are different in terms of availability of partial information: (1) the finite range $[0, b]$ and the mode $m$ of the demand during lead-time are known; and (2) the finite range $[0, b]$, the mode $m$ and the expected value $m_{1}$ of the demand during lead-time are known.

\section{THE DIRECT PROBLEM}

In this section on the direct problem, we aim to compare the upper and lower bounds obtained in the case of two additional types of information to the mean, i.e. (1) the mode in addition to the mean, and (2) the variance in addition to the mean. The first subsection investigates briefly the influence of the knowledge of the mode only. It serves as comparison with results of the second subsection.

\subsection{Unimodal demand distributions with known range and mode, no moments known}

The dual problem of this problem can be formulated, similar to (3.9), as:

$$
Q\left(z^{\prime}\right)=\inf \left(y_{3} \mid y_{3} \geq \hat{f}_{3}(\theta)\right)
$$

where the values of $\hat{f}_{3}(\theta)$ are calculated from equation (4.9) for $m<t$. In this case no linear program needs to be solved as the maximum value of $\hat{f}_{3}(\theta)$ is the solution to the upper bound problem.

As $m<t$, equation (4.9) is increasing in $x$, so its maximum value is obtained for $x=b$. The extreme distribution, associated with the upper bound, is a uniform distribution on $[m, b]$. The extreme distribution, associated with the lower bound, can, for example, be the uniform distribution on $[0, m]$, which means the lower bound is equal to zero. 
TABLE 4. Data for the case no moments known and $m<t$.

\begin{tabular}{ll}
\hline \hline$m$ & 5,15 \\
{$[a, b]$} & {$[0,50]$} \\
$t$ & 10,25 \\
\hline
\end{tabular}

TABLE 5. Upper and lower bounds on the number of units short.

\begin{tabular}{lll}
\hline \hline & Upper & Lower \\
\hline$t=10(m=5)$ & 17.7778 & 0.0000 \\
$t=25(m=15)$ & 8.9286 & 0.0000 \\
\hline
\end{tabular}

TABle 6. Data for the case with expected value known and $m<t$.

\begin{tabular}{ll}
\hline \hline$m_{1}$ & 25 \\
$m$ & 5 \\
{$[a, b]$} & {$[0,50]$} \\
Number of approximation points $(k)$ & $10,20,40,80$ \\
$t$ & 10 \\
\hline
\end{tabular}

TABLE 7. Upper and lower bounds on the number of units short.

\begin{tabular}{lcccc}
\hline \hline & $k=10$ & $k=20$ & $k=40$ & $k=80$ \\
\hline Upper bounds & 16.0000 & 16.0000 & 16.0000 & 16.0000 \\
Lower bounds & 15.3125 & 15.3125 & 15.3125 & 15.3125 \\
\hline
\end{tabular}

Example 5.1. The data for the first illustration are given in Table 4. The objective function values are shown in Table 5. The interval is the same as in Examples 3.1 and 3.2. Two values of the reorder point $t=10$ and $t=25$ are chosen from Example 3.1.

The results in Table 4 are not comparable to those in Table 2 as both optimisation problems use different information on the demand. Table 2 uses the mean and variance and Table 4 uses the mode.

\subsection{Unimodal demand distributions with known range, expected value and mode}

This section, compared to Section 5.1, adds information on the expected value of the demand distribution, next to range and mode.

Example 5.2. The data for the second illustration are given in Table 6. The objective function values, as obtained through the dual linear programs, are shown in Table 7.

Due to the additional constraint on the expected value the upper bounds are lower than or equal to the values in Table 5, and the lower bounds are higher than or equal to the values in Table 5. Note that the value of the number of discrete points in the approximation, $k$, is not of importance in this case, so, already for $k=10$, the optimal values of the bounds are obtained. But this phenomenon observed cannot be generalised. 
TABLE 8. Data for the case with expected value and variance known.

\begin{tabular}{ll}
\hline \hline$m_{1}$ & 25 \\
$m_{2}$ & 725 \\
{$[a, b]$} & {$[0,50]$} \\
Number of approximation points $(k)$ & $10,20,40,80$ \\
$t$ & 10 \\
\hline
\end{tabular}

TABLE 9. Upper and lower bounds on the number of units short.

\begin{tabular}{lllll}
\hline \hline & $k=10$ & $k=20$ & $k=40$ & $k=80$ \\
\hline Upper bounds & 16.3333 & 16.3636 & 16.3768 & 16.3784 \\
Lower bounds & 15.0000 & 15.0000 & 15.0000 & 15.0000 \\
\hline
\end{tabular}

\subsection{Unimodal demand distributions with known range, expected value and variance}

In this section another type of additional information is taken into consideration, i.e. the variance instead of the mode. In such a way a comparison study on specific cases can be made whether knowledge of the mode or the variance offers most additional information to the knowledge of the expected value.

Example 5.3. The data for the third illustration are given in Table 8. The objective function values as obtained through the dual linear programs are shown in Table 9.

Comparing Tables 7 and 9, it can be stated that the mode contains more information than the variance as both upper and lower bounds are closer to each other. This observation, however, cannot be generalised but the technique proposed, at least, allows for this type of evaluation.

\section{THE INVERSE PROBLEM}

\subsection{Unimodal demand distributions with known range, expected value and mode}

Similar to Section 2, the decision might be formulated to answer the following question: given a maximum expected number of units short, what should be the safety inventory at least (or at most)? In this section the focus is put only on the option "at most", which is the relevant option for the industry. However, in the case of unimodal distributions, with known unique mode, some transformations need to be included in the nonlinear optimisation model.

In comparison with the development in Section 2, the objective function (3.12) and constraint (3.13) remain unchanged as a solution of points for which the probability mass is looked for. Constraint (3.14) requires a change as, through the transformation, the expected value on the right hand side of the equality is no longer valid. The transformed variable $Y$ in Section 3 has expected value:

$$
E(Y)=\nu_{1}=2 \times m_{1}-m .
$$

Constraint (3.15) is not of relevance in this section as we consider only the knowledge of the mode and the first moment of the demand distribution. Also constraint (3.16) requires a change. The function $\left(x_{i}-t\right)_{+}$needs to be replaced by (4.9).

Example 6.1. The approach for unimodal distributions with known finite range, unique mode and expected value is illustrated by means of an example, with data shown in Table 10. For this example, the same range and expected value of the examples in the previous sections are chosen. 
TABLE 10. Data for the case with given maximal expected number of units short.

\begin{tabular}{ll}
\hline \hline$m_{1}$ & 25 \\
$m$ & 32 \\
{$[a, b]$} & {$[0,50]$} \\
Number of approximation points $(k)$ & 80 \\
Maximal expected number of units short $\left(z_{3}\right)$ & 2.25 \\
\hline
\end{tabular}

In the optimisation model, it is assumed that $m<t$ (which is, for practice, the more realistic case). The non-linear optimisation model to be solved is formulated as:

$$
[P 2] \text { Min } t \text {, }
$$

subject to

$$
\begin{aligned}
\sum_{i} p_{i} & =1, \\
\sum_{i} x_{i} \times p_{i} & =\nu_{1}, \\
\sum_{i} \frac{1}{2} \frac{\left(\left(x_{i}-t\right)_{+}\right)^{2}}{x_{i}-m} \times p_{i} & \leq z_{3} .
\end{aligned}
$$

Note that, due to equation (6.1), $\nu_{1}=18$, and that, similar to the procedure followed in Section 2 , the non-linear constraint (6.5) may be approximated by letting the value of $t$ coincide with one of the $x_{i}$-values. Also here a binary variable is introduced to indicate the condition " $t=x_{j}$ ", expressed as:

$$
\begin{aligned}
y_{j} & =1 \text { if } t=x_{j}, \\
\text { else } & =0 .
\end{aligned}
$$

The construction of the linear program is illustrated in Appendix A by means of a small example (with $k=11$ ). The non-linear constraint (6.5) is replaced by 11 constraints, indicating which of the $x_{j}$-points coincides with $t$. So (6.5) is replaced by:

$$
\sum_{i} \frac{1}{2} \frac{\left(\left(x_{i}-x_{j}\right)_{+}\right)^{2}}{x_{i}-32} \times p_{i} \leq 2.25 \times y_{j}+18 \times\left(1-y_{j}\right),
$$

with $j=0 \ldots 10$ and $x_{j}=5 \times j$. Appendix A shows a matrix with the coefficients at the left hand side of inequality (6.6). Further it shows the Lingo code in expanded form for the linear program of the example. The program results in a value $t=35$. In the next sections, the sensitivity of this value to varying values of the mode $m$ and the upper bound of the demand interval $b$ are investigated.

\subsection{Sensitivity of the reorder level to the mode}

As this paper deals explicitly with unimodal demand distributions, the knowledge of the mode is of high importance. From an inventory practical viewpoint, the mode needs to be estimated. While the mode has the advantage of being the least biased (compared to mean and median), it is more difficult to calculate than the mean and the median. For discrete data involving a relatively small number of possible values, the mode is easily calculated as the most frequent value. For continuous data, the methods by [6] and by [14] have been proposed. The former method is sensitive to the size of the interval selected, and the latter method is sensitive to outliers. These findings make it useful to check the impact of a wrong estimate of the mode on the decision on safety stock. 


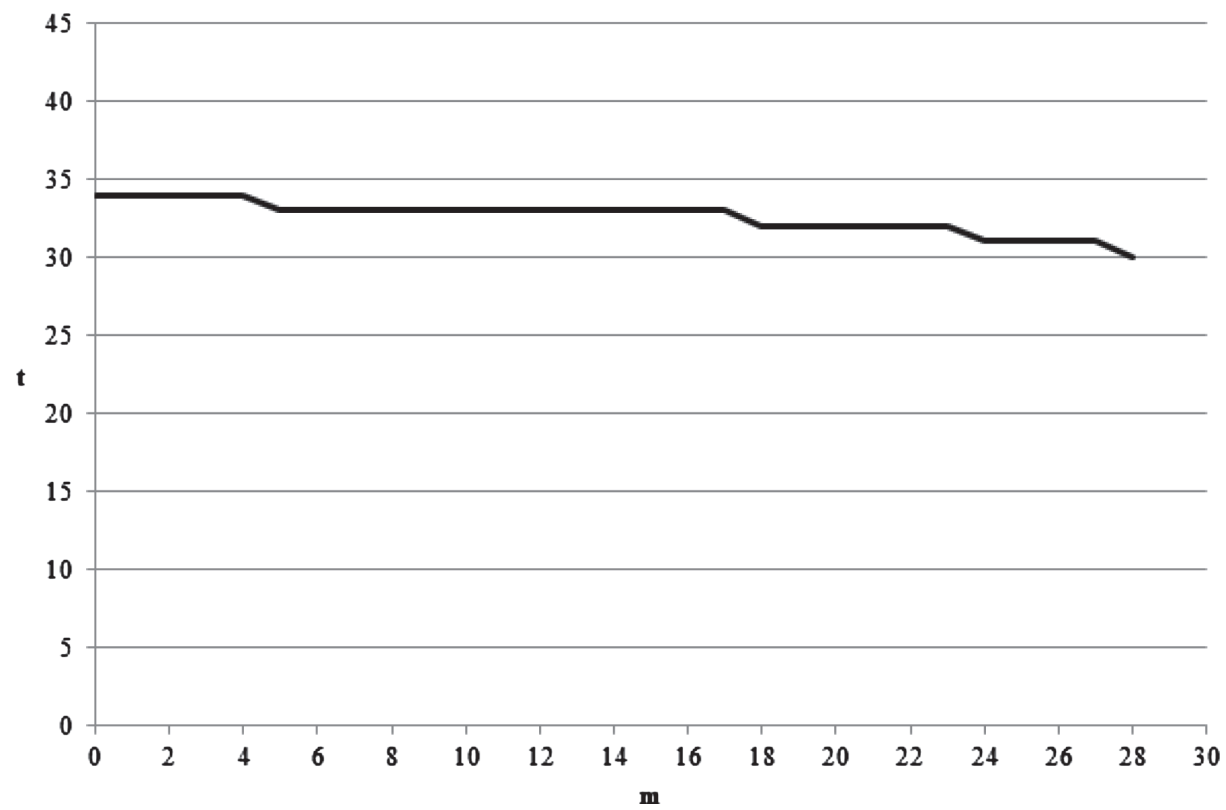

Figure 1. Sensitivity of the reorder level $t$ to the mode value $m$.

First, note that the determination of $t$ is independent of the mode $m$ in case the unknown distribution is symmetric. This is the case in our example from Table 9: the range is $[0,50]$ and the expected value equals 25 . In this simple case, the expected value of number of units short can be determined in an analytical way as:

$$
z_{3}=\frac{1}{2} \frac{(b-t)^{2}}{b} .
$$

The value of the mode $m$ does not appear in equation (6.7).

Example 6.2. As an illustration, the data from previous Section 6.1 in Table 10 are used, but sensitivity is explored against the values of the mode $m$. The risk towards the decision-maker exists in the fact that $m$ might be smaller than estimated. The exploration of the sensitivity towards $m$ leads to a horizontal line in Figure 1 as $t=35$ is sufficient, independent of $m$. For illustrative purposes, Figure 1 also shows the sensitivity towards $m$ for an asymmetrical distribution (expected value equals 20, $z_{3}$ remains 2.25 as in Tab. 9). Figure 1 shows the required reorder level $t$ in function of the values of $m$, ranging from 28 downwards to the value 0 . The range in the experiment is extremely large but shows that the required reorder level hardly ranges from 30 till 34 . This fact illustrates that the qualitative aspect of having a unimodal distribution for the demand during lead-time is of greater importance than the knowledge of the modal value.

\subsection{Sensitivity of the reorder level to the upper bound of the demand distribution interval}

Many inventory studies in an environment of uncertainty make use of distributions defined on the positive real axis $[0,+\infty)$. The developments in this research make use of a finite interval $[0, b]$ with $b>0$. As the upper bound of such a distribution defined on a finite interval is hard to estimate, in this section a sensitivity analysis is performed on the influence of the value of the upper bound $b$. Figure 2 shows the results of the optimisation model for varying values of the upper bound $b$ in combination with varying values of the mode $m$ as some 


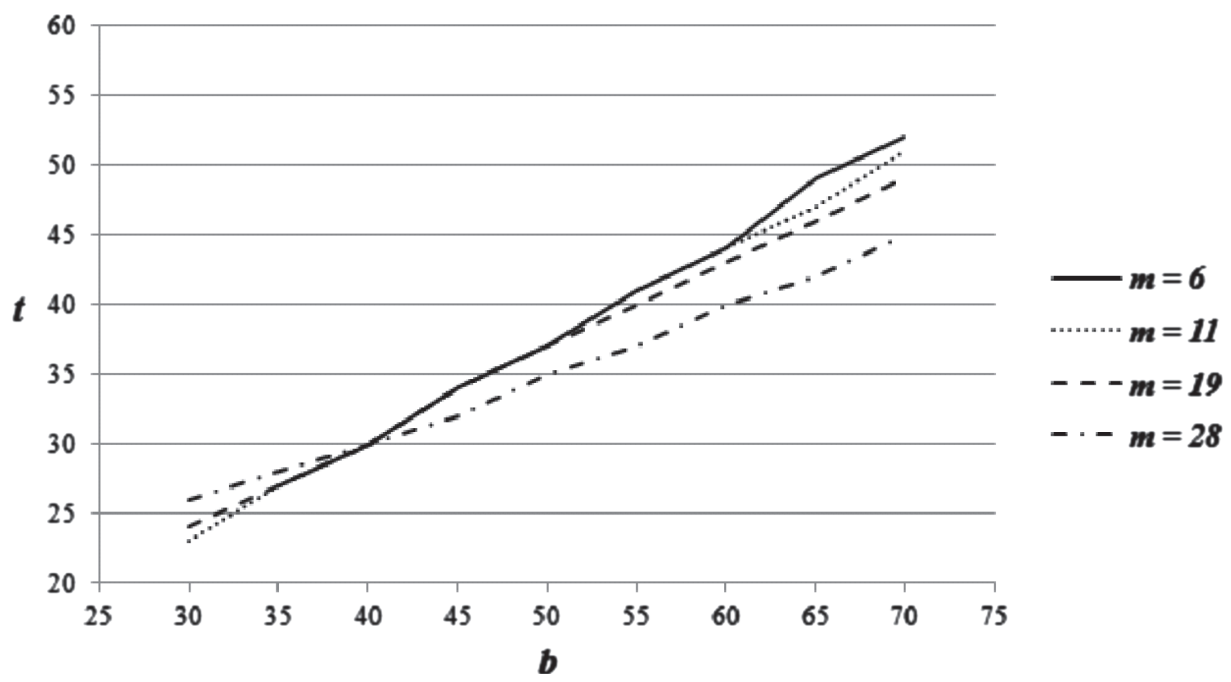

Figure 2. Sensitivity of the reorder level $t$ to the upper bound of the demand interval $b$.

interaction between both parameters might be expected. Also, here, the case of an asymmetric distribution (with expected value equal to 20) is chosen. In the case a symmetric distribution was imposed (expected value equal to 25) only a single line could be shown as the function between $t$ and $b$ is independent of $m$.

Figure 2 shows an increase of the reorder level in function of increasing values of the upper bound $b$, which is to be expected, but the slope of the function is different according to the value of the mode $m$. More specifically, it can be observed that the slope increases with lower values of the mode. In the extreme distributions, for smaller values of the mode, most part of the probability mass is assigned between the mode and the upper bound.

\section{Simulation StUdy MAKing USE OF THE DEVEloped METHOdOLOGY}

In this section a simulated environment is developed and illustrated for the theory developed in the previous sections. It is the aim of this section to start from a set of demand data, as a manager would be confronted with, and then apply the technique with various types of partial information. In the first subsection, the generation of the data for the Demand During Lead-Time (DDLT) is explained and how the relevant parameters for our method are determined. In the second subsection, the results of the optimization models, with varying partial information, are presented. This comparison might give a view on the relevance of pieces of distribution information like range, moments and/or mode.

\subsection{Data generation}

The series of DDLT data are generated by a simulation method. DDLT data are generated, following a triangular distribution. The range of the distribution equals $[0,50]$ as in the examples in the previous sections. The mode of the triangular distribution is chosen as $(15,25,35)$ to mimic a left-asymmetric, symmetric and right-symmetric triangular distribution. To include a sense of real-life, a not-too-long, series is generated (in this case, arbitrarily set at 20 periods) in order to avoid a theoretical long-term distribution but allowing for characteristics of small samples. In order to make meaningful conclusions on the simulation results, a number of stochastic replications of the same experimental setting are done (in this case, arbitrarily set at 5 replications).

In order to offer full reproduction of our simulation results, the procedure of generating the demand data, based on triangular distributions, is explained in detail. The generation for the triangular demand data is based 
TABLE 11. Symmetric triangular distribution.

\begin{tabular}{llllll}
\hline \hline Replication & 1 & 2 & 3 & 4 & 5 \\
\hline$Z_{0}$ & 6953867 & 9576389 & 48475613 & 3169483 & 71777548 \\
Range & 44.74 & 38.97 & 42.61 & 41.82 & 42.63 \\
First moment & 24.71 & 26.87 & 25.96 & 26.08 & 26.67 \\
Second moment & 698.73 & 783.62 & 768.65 & 753.37 & 785.77 \\
Mode & 26.92 & 22.43 & 23.75 & 22.28 & 27.08 \\
\hline
\end{tabular}

TABLE 12. Left-asymmetric triangular distribution.

\begin{tabular}{llllll}
\hline \hline Replication & 1 & 2 & 3 & 4 & 5 \\
\hline$Z_{0}$ & 6953867 & 9576389 & 48475613 & 3169483 & 71777548 \\
Range & 43.77 & 36.95 & 41.25 & 42.71 & 41.28 \\
First moment & 21.17 & 23.22 & 22.53 & 21.49 & 23.09 \\
Second moment & 544.08 & 610.37 & 612.61 & 602.80 & 617.67 \\
Mode & 20.59 & 16.33 & 19.27 & 19.03 & 22.88 \\
\hline
\end{tabular}

on generated data from a uniform distribution $[0,1]$. The latter data are generated by a Linear Congruential Generator (LCG) of the type

$$
Z_{i}=\left(a Z_{i-1}+c\right) \bmod m
$$

where $m$ is the modulus, $a$ the multiplier, $c$ the increment and $Z_{0}$ the seed or starting value.

Note that the symbols $m, a$ and $c$ should not be confused with the same symbols in the previous sections, but these symbols are classical in the simulation literature, which explains why they are used here. Many such LCG's have been proposed and tested. In this case, a widely used generator, which has been proposed in the Borland $C^{++}$-package, is used. It has the following parameter values: $m=2^{32}, a=22695477$, and $c=1$. For the 5 replications, the following values of the seed $Z_{0}$ are chosen from the vector $\mathbf{Z}_{\mathbf{0}}=(6953867,9576389$, $48475613,3169483,71777548$ ). The LCG generates integer values between 0 and $2^{32}-1$, so by dividing this integer value by its maximal attainable value, a variate $U$ is obtained, which follows a uniform distribution in the interval $[0,1]$. Given the values of $U$, the triangular distributed values can be obtained by:

$$
\begin{aligned}
X & =a+\sqrt{U(b-a)(m-a)} & & \text { for } 0<U<F(m) \\
& =b-\sqrt{(1-U)(b-a)(b-m)} & & \text { for } F(m) \leq U<1
\end{aligned}
$$

where $F(m)=(m-a) /(b-a)$.

The parameter values, required for the application of the methods developed in the previous sections, are obtained as follows: (1) the range: lower bound is zero and upper bound is the maximum value obtained from the data; (2) the first moment: obtained as the sample mean from the data; (3) the second moment: obtained from the sample mean and the sample variance from the data; and (4) the mode, as calculated by a method by Dalenius [6]. Those parameters are shown in Table 11 for the symmetric distribution, Table 12 for the left-asymmetric distribution, and Table 13 for the right-symmetric distribution.

\subsection{Results of the optimization procedure}

Given the parameters, obtained in the previous subsection, the optimization is run to obtain the decision values for the re-order points. The value of the maximum expected value of the units short $\left(z_{3}\right)$ is chosen equal to 2.25 (like in Sect. 6). Three tables are shown to present the results for the symmetric, left-asymmetric and 
TABLE 13. Right-asymmetric triangular distribution.

\begin{tabular}{llllll}
\hline \hline Replication & 1 & 2 & 3 & 4 & 5 \\
\hline$Z_{0}$ & 6953867 & 9576389 & 48475613 & 3169483 & 71777548 \\
Range & 45.92 & 41.46 & 44.27 & 45.23 & 44.29 \\
First moment & 28.23 & 30.58 & 29.40 & 27.72 & 30.32 \\
Second moment & 888.35 & 997.46 & 960.61 & 903.33 & 993.76 \\
Mode & 31.62 & 32.51 & 31.94 & 25.06 & 31.80 \\
\hline
\end{tabular}

TABLE 14. Reorder points for the symmetric distribution cases.

\begin{tabular}{llllll}
\hline \hline Replication & 1 & 2 & 3 & 4 & 5 \\
\hline Normal approach & 28.22 & 28.83 & 29.83 & 28.73 & 29.40 \\
2-moment approach & 32.25 & 31.47 & 34.23 & 31.96 & 32.69 \\
1-moment + mode approach & 32.11 & 29.34 & 31.28 & 30.72 & 31.97 \\
\hline
\end{tabular}

TABLE 15. Reorder points for the left-asymmetric distribution cases.

\begin{tabular}{llllll}
\hline \hline Replication & 1 & 2 & 3 & 4 & 5 \\
\hline Normal approach & 25.11 & 25.75 & 26.96 & 27.75 & 26.39 \\
2-moment approach & 29.56 & 34.51 & 38.38 & 40.37 & 38.87 \\
1-moment + mode approach & 29.35 & 26.30 & 28.67 & 28.92 & 29.16 \\
\hline
\end{tabular}

TABLE 16. Reorder points for the right-asymmetric distribution cases.

\begin{tabular}{llllll}
\hline \hline Replication & 1 & 2 & 3 & 4 & 5 \\
\hline Normal approach & 31.92 & 32.58 & 33.36 & 33.68 & 33.05 \\
2-moment approach & 43.53 & 38.98 & 41.88 & 42.88 & 41.86 \\
1-moment + mode approach & 35.01 & 33.83 & 34.71 & 33.61 & 35.00 \\
\hline
\end{tabular}

right-asymmetric cases. The tables present the re-order points for the five replications as calculated in three ways. The first way, called the "Normal approach", uses the classical formula to obtain the safety stock or the re-order point, assuming the DDLT follows a normal distribution and mean and standard deviation are known (in this case from sample values). For this way, no linear program is required. The second way, called the "2-moment approach", uses the sample first and second moments to obtain the re-order point by a linear program, as developed in the previous sections. The third way, called the "1-moment + mode approach", uses the sample first moment and the mode estimation, as described in Appendix C, to obtain the re-order point by a linear program, as developed in the previous sections.

A first observation, which can be made from Tables 14-16, is that the level of conservativeness is different for the 2-moment and the 1-moment + mode approach. Both approaches are conservative as they look in a set of distributions, with specific characteristics, for the worst case. In all tables, for all replications, the re-order point for the 1-moment + mode approach is lower than the one proposed by the 2 -moment approach. It means that the knowledge of the mode is worth more than the knowledge of the variance. And this, even if the range of estimates of the mode is quite large. A second observation relates to the range of the proposed values for 
the re-order point. For the symmetric distribution cases, the ranges are nearly equal: 2.76 (34.23-31.47) versus 2.77 (32.11-29.34). But for the asymmetric cases, the ranges are much more different. For the left-asymmetric distribution cases, the range for the 2-moment approach equals $10.81(40.37-29.56)$ and, for the 1-moment + mode approach, it equals 3.05 (29.35-26.30). For the right-asymmetric distribution cases, the range for the 2-moment approach equals $4.55(43.53-38.98)$ and, for the 1 -moment + mode, it equals 1.40 (35.01-33.61). In both asymmetric cases, also the range for the 1-moment + mode approaches has the smaller range.

\section{Conclusions}

This work studies inventory management in an environment of uncertainty in demand. More specifically, distributions of demand during lead time with partial information are considered. Unimodal distributions are studied for which the range, the expected value, and the unique mode are known.

Given a service target for the inventory manager, defined as the average number short per replenishment cycle, it is shown how a nonlinear optimisation model can be formulated to answer the question of the required minimum reorder level, which leads the expected number of units short at most. Furthermore, a technique is proposed how to approximate the nonlinear model by a linear programming model. Experiments are run to investigate the influence of the number of evaluation points on the quality of the approximation.

Finally, the sensitivity of the values of the mode and of the upper bound of the finite demand distribution interval are tested. The experiments show that a reasonable misestimation of the mode is not of great importance. More attention should be paid to the estimation of the upper bound of the demand distribution interval, especially in combination with the knowledge of the mode.

\section{Appendix A. Code for Example 6.1}

Expanded Lingo code for the linear program with $m_{1}=25, m=32$ and $z_{3}=2.25$. As the Lingo code requires all terms, including a decision variable, to appear on the left side, equation (6.6) takes the form:

$$
\sum_{i} \frac{1}{2} \frac{\left(\left(x_{i}-x_{j}\right)_{+}\right)^{2}}{x_{i}-32} \times p_{i}-2.25 \times y_{j}+18 \times y_{j} \leq 18 .
$$

(1) $\quad M I N=T+1000 \times S_{1}+1000 \times S_{2}+1000 \times S_{3}+1000 \times S_{4}+1000 \times S_{5}+1000 \times S_{6}+1000 \times S_{7}+1000 \times$ $S_{8}+1000 \times S_{9}+1000 \times S_{10}+1000 \times S_{11}$

(2) $P_{1}+P_{2}+P_{3}+P_{4}+P_{5}+P_{6}+P_{7}+P_{8}+P_{9}+P_{10}+P_{11}=1 ;$

(3) $5 \times P_{2}+10 \times P_{3}+15 \times P_{4}+20 \times P_{5}+25 \times P_{6}+30 \times P_{7}+35 \times P_{8}+40 \times P_{9}+45 \times P_{10}+50 \times P_{11}=18$;

(4) $\quad-0.46296 \times P_{2}-2.27273 \times P_{3}-6.61764 \times P_{4}-16.6667 \times P_{5}-44.6429 \times P_{6}-225 \times P_{7}+204.167 \times P_{8}+$ $100 \times P_{9}+77.8846 \times P_{10}+69.4444 \times P_{11}+15.75 \times Y_{1}+S_{1}=18$

(5) $\quad-0.56818 \times P_{3}-2.94118 \times P_{4}-9.375 \times P_{5}-28.5714 \times P_{6}-156.25 \times P_{7}+150 \times P_{8}+76.5625 \times P_{9}+$ $61.5385 \times P_{10}+56.25 \times P_{11}+15.75 \times Y_{2}+S_{2}=18$

(6) $\quad-0.735294 \times P_{4}-4.16667 \times P_{5}-16.0714 \times P_{6}-100 \times P_{7}+104.167 \times P_{8}+56.25 \times P_{9}+47.1154 \times P_{10}+$ $44.4444 \times P_{11}+15.75 \times Y_{3}+S_{3}=18$;

(7) $\quad-1.04167 \times P_{5}-7.14286 \times P_{6}-56.25 \times P_{7}+66.66667 \times P_{8}+39.0625 \times P_{9}+34.6154 \times P_{10}+34.0278 \times$ $P_{11}+15.75 \times Y_{4}+S_{4}=18$

(8) $\quad-1.785714 \times P_{6}-25 \times P_{7}+37.5 \times P_{8}+25 \times P_{9}+24.0385 \times P_{10}+25 \times P_{11}+15.75 \times Y_{5}+S_{5}=18$;

(9) $\quad-6.25 \times P_{7}+16.6667 \times P_{8}+14.0625 \times P_{9}+15.3846 \times P_{10}+17.3611 \times P_{11}+15.75 \times Y_{6}+S_{6}=18$;

(10) $4.16667 \times P_{8}+6.25 \times P_{9}+8.653846 \times P_{10}+11.1111 \times P_{11}+15.75 \times Y_{7}+S_{7}=18$;

(11) $1.5625 \times P_{9}+3.84615 \times P_{10}+6.25 \times P_{11}+15.75 \times Y_{8}+S_{8}=18$;

(12) $0.961538 \times P_{10}+2.77778 \times P_{11}+15.75 \times Y_{9}+S_{9}=18$;

(13) $\quad 0.694444 \times P_{11}+15.75 \times Y_{10}+S_{10}=18$;

(14) $15.75 \times Y_{11}+S_{11}=18$;

(15) $T>=0$; 
(16) $T-5 \times Y_{2}>=0$;

(17) $T-10 \times Y_{3}>=0$;

(18) $T-15 \times Y_{4}>=0$;

(19) $T-20 \times Y_{5}>=0$;

(20) $T-25 \times Y_{6}>=0$;

(21) $T-30 \times Y_{7}>=0$;

(22) $T-35 \times Y_{8}>=0$;

(23) $T-40 \times Y_{9}>=0$;

(24) $T-45 \times Y_{10}>=0$;

(25) $T-50 \times Y_{11}>=0$;

(26) $Y_{1}+Y_{2}+Y_{3}+Y_{4}+Y_{5}+Y_{6}+Y_{7}+Y_{8}+Y_{9}+Y_{10}+Y_{11}=1$;

$@ B I N\left(Y_{1}\right) ; @ B I N\left(Y_{2}\right) ; @ B I N\left(Y_{3}\right) ; @ B I N\left(Y_{4}\right) ; @ B I N\left(Y_{5}\right) ; @ B I N\left(Y_{6}\right) ; @ B I N\left(Y_{7}\right)$;

$@ B I N\left(Y_{8}\right) ; @ B I N\left(Y_{9}\right) ; @ B I N\left(Y_{10}\right) ; @ B I N\left(Y_{11}\right)$;

END

TABLE A.1. Coefficients $x_{i}$ and $x_{j}$ for inequality (6.6).

\begin{tabular}{lllllll}
\hline \hline$x_{i} / x_{j}$ & 0 & 5 & 10 & 15 & 20 & 25 \\
\hline 0 & 0 & -0.4629 & -2.2727 & -6.6176 & -16.666 & -44.642 \\
5 & 0 & 0 & -0.5681 & -2.9411 & -9.375 & -28.571 \\
10 & 0 & 0 & 0 & -0.7352 & -4.1666 & -16.071 \\
15 & 0 & 0 & 0 & 0 & -1.0416 & -7.1428 \\
20 & 0 & 0 & 0 & 0 & 0 & -1.7857 \\
25 & 0 & 0 & 0 & 0 & 0 & 0 \\
30 & 0 & 0 & 0 & 0 & 0 & 0 \\
35 & 0 & 0 & 0 & 0 & 0 & 0 \\
40 & 0 & 0 & 0 & 0 & 0 & 0 \\
45 & 0 & 0 & 0 & 0 & 0 & 0 \\
50 & 0 & 0 & 0 & 0 & 0 & 0 \\
\hline
\end{tabular}

TABle A.2. Coefficients $x_{i}$ and $x_{j}$ for inequality (6.6).

\begin{tabular}{llllll}
\hline \hline$x_{i} / x_{j}$ & 30 & 35 & 40 & 45 & 50 \\
\hline 0 & -225 & 204.1667 & 100 & 77.88462 & 69.44444 \\
5 & -156.25 & 150 & 76.5625 & 61.53846 & 56.25 \\
10 & -100 & 104.1667 & 56.25 & 47.11538 & 44.44444 \\
15 & -56.25 & 66.66667 & 39.0625 & 34.61538 & 34.02778 \\
20 & -25 & 37.5 & 25 & 24.03846 & 25 \\
25 & -6.25 & 16.66667 & 14.0625 & 15.38462 & 17.36111 \\
30 & 0 & 4.166667 & 6.25 & 8.653846 & 11.11111 \\
35 & 0 & 0 & 1.5625 & 3.846154 & 6.25 \\
40 & 0 & 0 & 0 & 0.961538 & 2.777778 \\
45 & 0 & 0 & 0 & 0 & 0.694444 \\
50 & 0 & 0 & 0 & 0 & 0 \\
\hline
\end{tabular}




\section{Appendix B. Notation}

The notation used throughout the paper is shown in Table B.1.

TABLE B.1. Notation.

\begin{tabular}{|c|c|}
\hline$X$ & $\begin{array}{l}\text { Stochastic demand during lead-time, with } F(x) \text { as probability distribution } \\
\text { function, } f(x) \text { as probability density function, } m_{1}=E[X] \text {, and } m_{2}=E\left[X^{2}\right]\end{array}$ \\
\hline$t$ & Inventory reorder point level \\
\hline$Q$ & Fixed order quantity \\
\hline$I=[a, b]$ & $\begin{array}{l}\text { Finite support interval of the demand during lead-time distribution } \\
\text { with } a \geq 0 \text { and } a<b\end{array}$ \\
\hline$m$ & Mode of unimodal distribution \\
\hline$v_{1}$ & Transformed value of $m_{1}$ for unimodal distributions \\
\hline$n$ & Number of integral constraints in optimisation problem \\
\hline$z$ & Pre-set maximum expected number of units short \\
\hline$x_{i}$ & Discrete point in the interval $[a, b]$ \\
\hline$p_{i}$ & Probability mass in point $x_{i}$ \\
\hline
\end{tabular}

\section{Appendix C. Estimation of the mode}

As the estimation of the mode is far from popular - Dalenius [6] even calls it a neglected statistical parameter -, this appendix shows the procedure how the mode is estimated in the experiments from Section 7 . The procedure follows the estimator, called $M_{1}^{*}$ by Dalenius ([6], p. 113).

(1) Order the sample values of size $n$, so that $x_{1}<x_{2}<\ldots<x_{j}<\ldots<x_{j+k}<\ldots<x_{n}$.

(2) Compute the differences $z(j, k)=x_{j+k}-x_{j}$ for $j=1, \ldots, n-k$ and some integer $k<n$.

(3) Choose the shortest interval $\left(x_{j}, x_{j+k}\right)$, i.e. $\min z(j, k)$.

(4) Choose the mid-point of the interval as estimate of the mode.

Dalenius [6] gives no advice on the value of $k$. In his experiments he uses rather small values of $k$ but observes quite some variability using different $k$-values. Therefore, our approach is to estimate the mode using values $k=1 \ldots 5$, calculate the mode using the procedure and then averaging over the five values to obtain the value to be used in Section 7.2.

\section{REFERENCES}

[1] E. Bartezzaghi, R. Verganti and G. Zotteri, Measuring the impact of asymmetric demand distributions on inventories. Int. J. Prod. Econ. 60-61 (1999) 395-404.

[2] D. Bertsimas and A. Thiele, A robust optimization approach to inventory theory. Oper. Res. 54 (2006) $150-168$.

[3] W. Bruggeman, H. Muller and S. de Samblanckx, Evaluation of inventory control systems in practice. Tijd. Econ. Manage. 25 (1980) 19-39.

[4] W. Chen, J. Li and X. Jin, The replenishment policy of agri-products with stochastic demand in integrated agricultural supply chains. Expert Syst. App. 48 (2016) 55-66.

[5] J. Croston, Forecasting and stock control for intermittent demands. Oper. Res. Q. 23 (1972) 289-303.

[6] T. Dalenius, The mode - a neglected statistical parameter. J. R. Stat. Soc. (Ser. A) 128 (1965) 110-117.

[7] F. De Vylder and M. Goovaerts, Upper and lower bounds on stop-loss premiums in case of known expectation and variance of the risk variable. Mitt. Ver. Schweiz. Versicherungsmathematiker 1 (1982) 149-164.

[8] O. Dey and D. Chakraborty, Fuzzy period review system with fuzzy random variable demand. Eur. J. Oper. Res. 198 (2009) $113-120$.

[9] S. Dharmadhikari and K. Joag-Dev, Unimodality, Convexity, and Applications. Academic Press, Boston, MA (1988).

[10] W. Feller, An Introduction to Probability Theory and its Applications. Wiley and Sons, New York, NY (1971). 
[11] G. Gallego, A minimax distribution free procedure for the $(Q, R)$ inventory model. Oper. Res. Lett. 11 (1992) 55-60.

[12] P. Garthwaite, J. Kadane and A. O'Hagan, Statistical methods for eliciting probability distributions. J. Am. Stat. Assoc. 100 (2005) 680-701.

[13] M. Goovaerts, J. Haezendonck and F. De Vylder, Numerical best bounds on stop-loss premiums. Insur. Math. Econ. 1 (1982) $287-302$.

[14] U. Grenander, Some direct estimates of the mode. Ann. Math. Stat. 36 (1965) 131-138.

[15] R. Heuts, J. van Lieshout and K. Baken, An inventory model: what is the influence of the shape of the lead time demand distribution. Z. Oper. Res. 30 (1986) B1-B14.

[16] J. Hull, The accuracy of the means and standard deviations of subjective probability distributions. J. R. Stat. Soc. (Ser. A) 141 (1978) 79-85.

[17] G.K. Janssens and K. Ramaekers, On the use of bounds on the stop-loss premium for an inventory management decision problem. J. Interdiscip. Math. 11 (2008) 115-126.

[18] G.K. Janssens and K. Ramaekers, A linear programming formulation for an inventory management decision problem with a service constraint. Expert Syst. App. 38 (2011) 7929-7934.

[19] G.K. Janssens, K. Ramaekers and L. Verdonck, Linear programming models to support inventory decision-making in the case of incomplete information on demand during lead-time. East West J. Math. 15 (2013) 109-126.

[20] A. Käki, A. Salo and S. Talluri, Impact of the shape of demand distribution in decision models for operations management. Comput. Ind. 64 (2013) 765-775.

[21] D. Keefer and S. Bodily, Three-point approximations for continuous random variables. Manage. Sci. 29 (1983) 595-609.

$[22]$ B. Li, H.-W. Wang, J.-B. Yang, M. Guo and C. Qi, A belief-rule-based inventory control method under nonstationary and uncertain demand. Expert Syst. App. 38 (2011) 14997-15008.

[23] D. Malcolm, J. Roseboom, C. Clark and W. Fazar, Application of a technique for research and development program evaluation. Oper. Res. 7 (1959) 646-669.

[24] E. Naddor, Sensitivity to distributions in inventory systems. Manage. Sci. 24 (1978) 1769-1772.

[25] S. Nahmias, Production and Operations Analysis. Irwin, Homewood, IL (1993).

[26] G. Perakis and G. Roels, Regret in the newsvendor model with partial information. Oper. Res. 56 (2008) $188-203$.

[27] C. Perry and I. Greig, Estimating the mean and variance of subjective distributions in PERT and decision analysis. Manage. Sci. 21 (1975) 1477-1480.

[28] R. Rossi, S. Tarim, B. Hnich and S. Prestwich, Replenishment planning for stochastic inventory systems with shortage cost, edited by P. Van Hentenryck and L. Wolsey. In: Integration of AI and OR Techniques in Constraint Programming for Combinatorial Optimization Problems. Springer Berlin-Heidelberg, Berlin (2007) 229-243.

[29] H. Scarf, A min-max solution of an inventory problem, edited by K. Arrow, S. Karlin and H. Scarf. In: Studies in the Mathematical Methods of Inventory and Production. Stanford University Press, Redwood City, CA (1958) $201-209$.

[30] E.A. Silver, D.F. Pyke and R. Peterson, Inventory Management and Production Planning and Scheduling, 3rd edition. Wiley and Sons, New York, NY (1998).

[31] A. Syntetos and J. Boylan, The accuracy of intermittent demand estimates. Int. J. Forecast. 21 (2005) 303-314.

[32] A. Syntetos and J. Boylan, Demand forecasting adjustments for service-level achievement. IMA J. Manage. Math. 19 (2008) $175-192$.

[33] R. Zapata-Vazquez, A. O'Hagan and L. Soares Bastos, Eliciting expert judgments about a set of proportions. J. Appl. Stat. 41 (2014) 1919-1933. 